IFUM-694-FT

hep-th/0110031

\title{
The Asymptotic Dynamics of de Sitter Gravity in three Dimensions
}

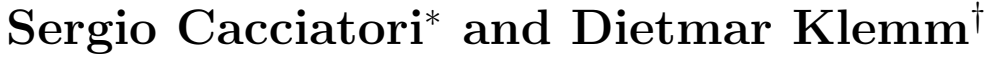

\author{
Dipartimento di Fisica dell'Università di Milano and \\ INFN, Sezione di Milano, Via Celoria 16, 20133 Milano, Italy.
}

\begin{abstract}
We show that the asymptotic dynamics of three-dimensional gravity with positive cosmological constant is described by Euclidean Liouville theory. This provides an explicit example of a correspondence between de Sitter gravity and conformal field theories. In the case at hand, this correspondence is established by formulating Einstein gravity with positive cosmological constant in three dimensions as an $\mathrm{SL}(2, \mathbb{C})$ Chern-Simons theory. The de Sitter boundary conditions on the connection are divided into two parts. The first part reduces the CS action to a nonchiral $\operatorname{SL}(2, \mathbb{C})$ WZNW model, whereas the second provides the constraints for a further reduction to Liouville theory, which lives on the past boundary of $\mathrm{dS}_{3}$.
\end{abstract}

*cacciatori@mi.infn.it

†dietmar.klemm@mi.infn.it 


\section{Introduction}

Recently there has been an increasing interest in gravity on de Sitter (dS) spacetimes [1 [8]. The motivation for this comes partially from recent astrophysical data indicating a positive cosmological constant [19], but apart from phenomenological aspects, it also remains an outstanding challenge to understand the role of de Sitter space in string theory, to clarify the microscopic origin of de Sitter entropy 20 25], and to study in which way the holographic principle [26, 27] is realized in the case of de Sitter gravity. Whereas string theory on anti-de Sitter spaces is known to have a dual description in terms of certain superconformal field theories [28], no such explicit duality was known up to now for dS spacetimes. Based on [1], where the first evidence for a dS/CFT correspondence was given, and on related ideas that appeared in [2, 4, 29], Strominger proposed recently a holographic duality relating quantum gravity on $\mathrm{dS}_{D}$ to a Euclidean conformal field theory residing on the past boundary $\mathcal{I}^{-}$of $\mathrm{dS}_{D}$ [9]. In particular, he considered threedimensional de Sitter gravity, and showed that it admits an asymptotic symmetry algebra consisting of two copies of Virasoro algebras with central charges $c=\tilde{c}=3 l / 2 G$, where $l$ is the $\mathrm{dS}_{3}$ curvature radius, and $G$ denotes Newton's constant?. This central charge was then rederived by other methods in [10], where also further evidence for a dS/CFT correspondence was given.

The purpose of the present paper is to show that in the case of $2+1$-dimensional Einstein gravity with positive cosmological constant, the dual CFT in question is Euclidean Liouville field theory? This provides an explicit example of a dS/CFT correspondence, and shows how the asymptotic symmetries of $\mathrm{dS}_{3}$ are realized in the dual conformal field theory. To establish this correspondence, we shall make use of the Chern-Simons formulation [33, 34] of pure gravity in $2+1$ dimensions, and we will closely follow the analogous work that has been done for anti-de Sitter space [35 39].

The remainder of this paper is organized as follows:

In the next section, we briefly review the Chern-Simons formulation of three-dimensional Einstein gravity with positive cosmological constant, and translate the de Sitter boundary conditions on the metric [9] in terms of the CS connection. In section 3, we show that the first part of these conditions implies the reduction of the Chern-Simons action to a nonchiral $\mathrm{SL}(2, \mathbb{C})$ WZNW model. In $\mathbb{1}$, we make use of the remaining boundary conditions in order to further reduce the WZNW model to Liouville field theory, which lives on the past boundary $\mathcal{I}^{-}$of $\mathrm{dS}_{3}$. The appendix contains our conventions.

\section{De Sitter Gravity as Chern-Simons Theory}

Pure $2+1$-dimensional gravity with positive cosmological constant $\Lambda=1 / l^{2}$ is described by the action

\footnotetext{
${ }^{1}$ This generalizes the result of Brown and Henneaux [30] to the case of positive cosmological constant.

${ }^{2}$ Cf. also [31, 32] for the appearance of the Liouville equation and Liouville action in slightly different contexts.
} 


$$
S=\frac{1}{16 \pi G} \int d^{3} x \sqrt{-g}(R-2 \Lambda)
$$

The equations of motion following from (2.1) admit the de Sitter solution

$$
d s^{2}=-\frac{l^{2}}{\tau^{2}} d \tau^{2}+\tau^{2} d z d \bar{z}
$$

An asymptotically de Sitter geometry [9] is one for which the metric behaves for $\tau \rightarrow \infty$ as

$$
\begin{aligned}
g_{z \bar{z}} & =\frac{\tau^{2}}{2}+\mathcal{O}(1) \\
g_{z z} & =\mathcal{O}(1) \\
g_{\tau \tau} & =-\frac{l^{2}}{\tau^{2}}+\mathcal{O}\left(\tau^{-4}\right) \\
g_{z \tau} & =\mathcal{O}\left(\tau^{-3}\right) .
\end{aligned}
$$

Past infinity $\mathcal{I}^{-}$is a spacelike cylinder parametrized by the coordinates $\phi \sim \phi+2 \pi$ and $y$, where we set $z=\phi+i y$. We denote this surface by $\Sigma$.

In what follows, we shall make essential use of the fact that $2+1$-dimensional de Sitter gravity can be formulated as an $\operatorname{SL}(2, \mathbb{C})$ Chern-Simons theory [33, 34], with action

$$
S=\frac{i s}{4 \pi} \int \operatorname{Tr}\left(A \wedge d A+\frac{2}{3} A \wedge A \wedge A\right)-\frac{i s}{4 \pi} \int \operatorname{Tr}\left(\bar{A} \wedge d \bar{A}+\frac{2}{3} \bar{A} \wedge \bar{A} \wedge \bar{A}\right)
$$

where, in our conventions (cf. appendix),

$$
s=-\frac{l}{4 G},
$$

and $A$ denotes an $\operatorname{SL}(2, \mathbb{C})$ gauge field. (2.1) and (2.4) are equivalent, if we identify

$$
A=A^{a} \tau_{a}=\left(\omega^{a}+\frac{i}{l} e^{a}\right) \tau_{a}, \quad \bar{A}=\bar{A}^{a} \tau_{a}=\left(\omega^{a}-\frac{i}{l} e^{a}\right) \tau_{a}, \quad a=0,1,2,
$$

\footnotetext{
${ }^{3}$ This is related to the planar slicing of $\mathrm{dS}_{3}$ given in 99 by setting $\tau=l \exp (-t)$. The past boundary $\mathcal{I}^{-}(t \rightarrow-\infty)$ corresponds then to $\tau \rightarrow \infty$. The Carter-Penrose diagram can be found in [9].
} 
where $e^{a}$ is the dreibein, $\omega^{a}=\frac{1}{2} \epsilon^{a b c} \omega_{b c}$ the $\operatorname{SL}(2, \mathbb{R})$ spin connection, and the $\tau_{a}$ are the $\mathrm{SL}(2, \mathbb{C})$ generators (cf. appendix).

Modulo total derivatives' the action (2.4) can be rewritten as

$$
S=S_{C S}[A]-S_{C S}[\bar{A}]
$$

with

$$
S_{C S}[A]=\frac{i s}{4 \pi} \int d^{3} x \operatorname{Tr}\left(\dot{A}_{\tau} A_{\phi}-\dot{A}_{\phi} A_{\tau}+2 A_{y} F_{\tau \phi}\right)
$$

where a dot denotes the derivative with respect to $y$, and $F=d A+A \wedge A$.

In terms of the connection $A$, the boundary conditions (2.3) read

$$
A=\left(\begin{array}{cc}
\frac{d \tau}{2 \tau} & \mathcal{O}(1 / \tau) \\
\frac{i \tau}{l} d z & -\frac{d \tau}{2 \tau}
\end{array}\right), \quad \bar{A}=\left(\begin{array}{cc}
-\frac{d \tau}{2 \tau} & -\frac{i \tau}{l} d \bar{z} \\
\mathcal{O}(1 / \tau) & \frac{d \tau}{2 \tau}
\end{array}\right)
$$

Like in the AdS case [35], we can state two essential points concerning the asymptotic behaviour (2.9) of the connection:

1. The components $A_{\bar{z}}$ and $\bar{A}_{z}$ are set to zero asymptotically.

2. $A_{z}^{+}=A_{z}^{1}+i A_{z}^{2}$ and $\bar{A}_{\bar{z}}^{-}=\bar{A}_{\bar{z}}^{1}-i \bar{A}_{\bar{z}}^{2}$ are independent of $z, \bar{z}$ to leading order in $\tau$. Also, $A_{z}^{0}$ and $\bar{A}_{\bar{z}}^{0}$ vanish.

In section 3, following [35, 40], we show that the first condition implies a reduction of the Chern-Simons action to a nonchiral WZNW model. The second condition then provides the constraints for a further reduction from the WZNW model to Liouville theory.

\section{Reduction to a WZNW Model}

When $A_{\bar{z}}$ and $\bar{A}_{z}$ are required to vanish on the boundary $\mathcal{I}^{-}=\Sigma$, the action (2.7) is not extremal on-shell. Instead, $\delta S$ equals the surface term $\delta\left[-\frac{s}{4 \pi} \int_{\Sigma} d \phi d y \operatorname{Tr}\left(A_{\phi}^{2}+\bar{A}_{\phi}^{2}\right)\right]$. In order to cancel this, we must add a boundary term to the action (2.7), leading to the improved action的

\footnotetext{
${ }^{4}$ Boundary terms will be considered below.

${ }^{5}$ Surface terms that arise at $y_{1}$ and $y_{2}$ will be discussed in section 4 . Besides, de Sitter space has two spacelike conformal boundaries $\mathcal{I}^{-}$and $\mathcal{I}^{+}$, so that in principle additional boundary terms at $\mathcal{I}^{+}$ have to be taken into account. However, since we are only interested in the asymptotic dynamics of the gravitational field near $\mathcal{I}^{-}$, we will ignore such surface terms. Some discussion of the problems that arise due to the presence of a second boundary can be found in [9], where it was argued that in spite of the fact that de Sitter space has two boundaries, the dual description of de Sitter gravity is provided by a single CFT.
} 


$$
S=S_{C S}[A]+\frac{s}{4 \pi} \int_{\Sigma} d \phi d y \operatorname{Tr}\left[\left(A_{\phi}\right)^{2}\right]-S_{C S}[\bar{A}]+\frac{s}{4 \pi} \int_{\Sigma} d \phi d y \operatorname{Tr}\left[\left(\bar{A}_{\phi}\right)^{2}\right]
$$

We recognize that $A_{y}$ and $\bar{A}_{y}$ are Lagrange multipliers that implement the Gauss law constraints $F_{\tau \phi}=\bar{F}_{\tau \phi}=0$. These are easily solved by

$$
A_{\mu}=G_{1}^{-1} \partial_{\mu} G_{1}, \quad \bar{A}_{\mu}=G_{2}^{-1} \partial_{\mu} G_{2}
$$

where $G_{1,2}$ have the asymptotic behaviour

$$
G_{1} \rightarrow g_{1}(z, \bar{z})\left(\begin{array}{cc}
\sqrt{\frac{\tau}{l}} & 0 \\
0 & \sqrt{\frac{l}{\tau}}
\end{array}\right), \quad G_{2} \rightarrow g_{2}(z, \bar{z})\left(\begin{array}{cc}
\sqrt{\frac{l}{\tau}} & 0 \\
0 & \sqrt{\frac{\tau}{l}}
\end{array}\right)
$$

and $g_{1,2}(z, \bar{z})$ are arbitrary elements of $\mathrm{SL}(2, \mathbb{C})$. Strictly speaking, the Gauss law constraints imply (3.2) only for $\mu=\tau, \phi$, whereas (3.2) for $\mu=y$ is a gauge choice. (3.3) implies the boundary condition (2.9) for $A_{\tau}$ and $\bar{A}_{\tau}$, whereas for the tangential components one obtains

$$
A_{j}=\left(\begin{array}{cc}
-\frac{i}{2} a_{j}^{0} & \frac{l}{2 \tau} a_{j}^{-} \\
\frac{\tau}{2 l} a_{j}^{+} & \frac{i}{2} a_{j}^{0}
\end{array}\right), \quad \bar{A}_{i}=\left(\begin{array}{cc}
-\frac{i}{2} \tilde{a}_{j}^{0} & \frac{\tau}{2 l} \tilde{a}_{j}^{-} \\
\frac{l}{2 \tau} \tilde{a}_{j}^{+} & \frac{i}{2} \tilde{a}_{j}^{0}
\end{array}\right)
$$

where $a_{j} \equiv g_{1}^{-1} \partial_{j} g_{1}, \tilde{a}_{j} \equiv g_{2}^{-1} \partial_{j} g_{2}$, and $j=z, \bar{z}$.

Inserting (3.2) into the action (3.1) yields a sum of two chiral WZNW models,

$$
S=S_{W Z N W}^{R}\left[g_{1}\right]-S_{W Z N W}^{L}\left[g_{2}\right]
$$

where

$$
\begin{aligned}
S_{W Z N W}^{R}\left[g_{1}\right] & =\frac{s}{2 \pi} \int d \phi d y \operatorname{Tr}\left[g_{1}^{-1} \partial_{\bar{z}} g_{1} g_{1}^{-1} \partial_{\phi} g_{1}\right]-\frac{i s}{12 \pi} \int \operatorname{Tr}\left(G_{1}^{-1} d G_{1}\right)^{3}, \\
S_{W Z N W}^{L}\left[g_{2}\right] & =-\frac{s}{2 \pi} \int d \phi d y \operatorname{Tr}\left[g_{2}^{-1} \partial_{z} g_{2} g_{2}^{-1} \partial_{\phi} g_{2}\right]-\frac{i s}{12 \pi} \int \operatorname{Tr}\left(G_{2}^{-1} d G_{2}\right)^{3} .
\end{aligned}
$$

These first order actions describe respectively a holomorphic group element $g_{1}(z)$ and an antiholomorphic group element $g_{2}(\bar{z})$. One has thus $a_{\bar{z}}=\tilde{a}_{z}=0$ on-shell, so that the first part of the boundary conditions is indeed satisfied.

\footnotetext{
${ }^{6}$ We did not consider possible holonomies, that appear as additional zero modes in $(3.2)$.
} 
The sum (3.5) of right- and left chiral actions is equivalent to the nonchiral WZNW action with dynamical variable $g=g_{1}^{-1} g_{2}$. To see this equivalence, we rewrite the action (3.5) in terms of the new variables $g$ and $\Pi \equiv-g_{2}^{-1} \partial_{\phi} g_{1} g_{1}^{-1} g_{2}-g_{2}^{-1} \partial_{\phi} g_{2}$. This leads to

$$
S=\frac{s}{2 \pi} \int d \phi d y \operatorname{Tr}\left[\frac{i}{2} \Pi g^{-1} \partial_{y} g+\frac{1}{4} \Pi^{2}+\frac{1}{4} g^{-1} \partial_{\phi} g g^{-1} \partial_{\phi} g\right]+\frac{i s}{12 \pi} \int \operatorname{Tr}\left(G^{-1} d G\right)^{3}
$$

with $G=G_{1}^{-1} G_{2}$. (3.7) is exactly the nonchiral WZNW model in first order formalism. Eliminating the auxiliary variable $\Pi$ by its equation of motion, one gets finally

$$
S=\frac{s}{2 \pi} \int d \phi d y \operatorname{Tr}\left[g^{-1} \partial_{z} g g^{-1} \partial_{\bar{z}} g\right]+\frac{i s}{12 \pi} \int \operatorname{Tr}\left(G^{-1} d G\right)^{3}
$$

which is the standard WZNW action.

\section{Further Reduction to Liouville Theory}

Up to now, we have implemented only part 1 of the boundary conditions on the ChernSimons connection. We must still incorporate the second part, which, in terms of the Kac-Moody currents, read

$$
J_{\bar{z}}^{-} \equiv\left(g^{-1} \partial_{\bar{z}} g\right)^{-}=-2 i, \quad \tilde{J}_{z}^{+} \equiv\left(\partial_{z} g g^{-1}\right)^{+}=-2 i
$$

and

$$
J_{\bar{z}}^{0}=\tilde{J}_{z}^{0}=0
$$

The constraints (4.1) are first class among themselves, and therefore generate a gauge symmetry, while the conditions (4.2) can be viewed as gauge conditions for the symmetry generated by (4.1) [37]. If one implements (4.1) and (4.2) by means of Lagrange multipliers, one gets the (gauged-fixed version of the) action for the gauged WZNW model, in which one has gauged the subgroup of $\operatorname{SL}(2, \mathbb{C})$ generated by the first class constraints [37. It is well known 41 44 that this model is equivalent to Liouville theory. To see this equivalence at the level of the action, we parametrize $g \in \operatorname{SL}(2, \mathbb{C})$ according to the Gauss decomposition

$$
g=\left(\begin{array}{cc}
1 & X \\
0 & 1
\end{array}\right)\left(\begin{array}{cc}
\exp \left(\frac{1}{2} \Phi\right) & 0 \\
0 & \exp \left(-\frac{1}{2} \Phi\right)
\end{array}\right)\left(\begin{array}{cc}
1 & 0 \\
Y & 1
\end{array}\right)
$$


where $X, Y, \Phi \in \mathbb{C}$. It will be shown below that actually $\Phi$ is real. With (4.3), the action (3.8) reads 43

$$
S=\frac{s}{2 \pi} \int d \phi d y\left[\frac{1}{2} \partial_{z} \Phi \partial_{\bar{z}} \Phi+2 \partial_{\bar{z}} X \partial_{z} Y \exp (-\Phi)\right] .
$$

In terms of the "momenta" $\Pi_{X}=\partial \mathcal{L} / \partial \dot{X}$ and $\Pi_{Y}=\partial \mathcal{L} / \partial \dot{Y}$ conjugate to $X, Y$, one obtains for the constraints (4.1)

$$
\Pi_{X}=\frac{i s}{2 \pi} \partial_{z} Y \exp (-\Phi)=\frac{s}{2 \pi}, \quad \Pi_{Y}=-\frac{i s}{2 \pi} \partial_{\bar{z}} X \exp (-\Phi)=-\frac{s}{2 \pi} .
$$

In order to implement (4.5), we have to go from (4.4) to the reduced action (Routhian function),

$$
S \rightarrow S-\int d \phi d y\left[\dot{X} \Pi_{X}+\dot{Y} \Pi_{Y}\right]
$$

i. e. we have to perform a partial Legendre transformation with respect to $\dot{X}, \dot{Y}$. This is equivalent to the procedure used in [35], which consists in adding a boundary term to (4.4), to get an improved action

$$
S_{i m p r}=S+\left.\frac{i s}{2 \pi} \oint d \phi\left[Y \partial_{\bar{z}} X-X \partial_{z} Y\right] \exp (-\Phi)\right|_{t_{1}} ^{t_{2}} .
$$

One can then insert (4.5) into (4.6) or (4.7), to obtain finally

$$
S=\frac{s}{2 \pi} \int d \phi d y\left[\frac{1}{2} \partial_{z} \Phi \partial_{\bar{z}} \Phi+2 \exp \Phi\right],
$$

which is the action of Euclidean Liouville field theory. We have thus shown that the asymptotic dynamics of three-dimensional de Sitter gravity is described by Liouville field theory. This provides an explicit example of the dS/CFT correspondence proposed by Strominger [9]. The two sets of Virasoro generators of Liouville theory are related to the residual Kac-Moody symmetries preserving the constraints (4.1) [35, 43], given by

$$
g(z, \bar{z}) \rightarrow \Omega(z) g(z, \bar{z}) \tilde{\Omega}^{-1}(\bar{z})
$$

with

$$
\Omega(z)= \pm\left(\begin{array}{cc}
1 & f(z) \\
0 & 1
\end{array}\right), \quad \tilde{\Omega}(\bar{z})= \pm\left(\begin{array}{cc}
1 & 0 \\
\tilde{f}(\bar{z}) & 1
\end{array}\right)
$$


where $f(z)(\tilde{f}(\bar{z}))$ is an arbitrary holomorphic (antiholomorphic) function.

We still have to show that the Liouville mode $\Phi$ is real. This is not evident from the Gauss decomposition (4.3), as this in general requires a complex $\Phi$ for the group $\operatorname{SL}(2, \mathbb{C})$. We did however not yet implement all the constraints. As our $\operatorname{SL}(2, \mathbb{C})$ generators satisfy $\tau_{a}^{\dagger}=\sigma \tau_{a} \sigma$ (cf. appendix), with $\sigma$ given in (A.5), the identification (2.6) leads to the pseudoreality condition

$$
A^{\dagger}=\sigma \bar{A} \sigma,
$$

which implies $G_{2}^{-1}=\sigma G_{1}^{\dagger} \tau$, where $\tau$ denotes an arbitrary constant element of $\operatorname{SL}(2, \mathbb{C})$. If we choose e. g. $\tau^{\dagger}=-\tau$, we obtain the relation】

$$
G^{\dagger}=-\sigma G \sigma,
$$

so that $G$ has the form

$$
G=\left(\begin{array}{cc}
u & w \\
-\bar{w} & v
\end{array}\right),
$$

with $u, v \in \mathbb{R}, w \in \mathbb{C}$. It is easy to see that (4.13) parametrizes an element of the coset space $\mathrm{SL}(2, \mathbb{C}) / \mathrm{SU}(2)$. The Gauss decomposition of (4.13) is given by (4.3) with $Y=-\bar{X}$ and $\Phi$ real. We can now impose this final constraint on the action (4.8) by means of a Lagrange multiplier $\lambda$, and then integrate in the path integral over $\lambda$ and over the imaginary part of $\Phi$, which leads to the same Liouville action, but with real $\Phi$. Alternatively, one can implement (4.12) already after the first reduction step, i. e. , one can restrict the group elements appearing in the WZNW action (3.8) to take values in the coset space $\mathrm{SL}(2, \mathbb{C}) / \mathrm{SU}(2)$. To this end, one first translates (1.12) into a constraint for the currents,

$$
J_{\bar{z}}^{\dagger}=-\sigma \tilde{J}_{z} \sigma,
$$

which is then imposed by means of a Lagrange multiplier in the action (3.8). This amounts to gauging the subgroup $\mathrm{SU}(2)$ of $\mathrm{SL}(2, \mathbb{C})$, generated by the constraints (4.14), i. e. , one obtains the action of the $\mathrm{SL}(2, \mathbb{C}) / \mathrm{SU}(2)$ gauged WZNW model. The integration constant $\tau$ relating $G_{1}$ and $G_{2}$ determines which subgroup is gauged. For example, the choice $\tau^{\dagger}=\tau$ leads to a gauging of the subgroup $\mathrm{SU}(1,1)$.

The classical Liouville solution corresponding to de Sitter space in horospherical coordinates (2.2) is given by

\footnotetext{
${ }^{7}$ The same holds for the boundary value $g$.
} 


$$
\exp \Phi=\left[-u z \bar{z}-i w z+i \bar{w} \bar{z}+\frac{1-w \bar{w}}{u}\right]^{-2}
$$

where $u \in \mathbb{R}$ and $w \in \mathbb{C}$ are arbitrary constants. By a combined dilation and translation, $u z \rightarrow z+i \bar{w}$, (4.15) can be cast in the elliptic form [45]

$$
\exp \Phi=\frac{u^{2}}{[1-z \bar{z}]^{2}}
$$

Possible further extensions of our work would be the consideration of holonomies, as well as the inclusion of the second boundary. In particular, it would be interesting to verify the argumentation of [9], that the holographic dual is a field theory on one boundary, rather than two. This is currently under investigation.

\section{Acknowledgements}

This work was partially supported by INFN, MURST and by the European Commission RTN program HPRN-CT-2000-00131, in which the authors are associated to the University of Torino.

\section{A Conventions}

Our $\operatorname{SL}(2, \mathbb{C})$ generators are

$$
\tau_{0}=\frac{1}{2}\left(\begin{array}{cc}
-i & 0 \\
0 & i
\end{array}\right), \quad \tau_{1}=\frac{1}{2}\left(\begin{array}{ll}
0 & 1 \\
1 & 0
\end{array}\right), \quad \tau_{2}=\frac{1}{2}\left(\begin{array}{cc}
0 & -i \\
i & 0
\end{array}\right) .
$$

They satisfy

$$
\left[\tau_{a}, \tau_{b}\right]=\epsilon_{a b c} \tau^{c}
$$

with $\epsilon_{012}=+1$, and are normalized according to

$$
\operatorname{Tr}\left(\tau_{a} \tau_{b}\right)=\frac{1}{2} \eta_{a b}
$$

where $\left(\eta_{a b}\right)=\operatorname{diag}(-1,1,1)$.

Another useful property is

$$
\tau_{a}^{\dagger}=\sigma \tau_{a} \sigma
$$


with $\sigma \in \mathrm{SL}(2, \mathbb{C})$ given by

$$
\sigma=\left(\begin{array}{cc}
i & 0 \\
0 & -i
\end{array}\right)
$$

We further define

$$
\tau_{ \pm}=\frac{1}{2}\left(\tau_{1} \mp i \tau_{2}\right) .
$$

Finally, $d \tau \wedge d \phi \wedge d y$ is chosen to have positive orientation. 


\section{References}

[1] C. M. Hull, "Timelike T-duality, de Sitter space, large N gauge theories and topological field theory," JHEP 9807 (1998) 021 [hep-th/9806146].

[2] C. M. Hull and R. R. Khuri, "Worldvolume theories, holography, duality and time," Nucl. Phys. B 575 (2000) 231 hep-th/9911082.

[3] T. Banks, "Cosmological breaking of supersymmetry," hep-th/0007146; T. Banks and W. Fischler, "M-theory observables for cosmological space-times," hepth/0102077].

[4] V. Balasubramanian, P. Horava and D. Minic, "Deconstructing de Sitter," JHEP 0105 (2001) 043 hep-th/0103171.

[5] E. Witten, "Quantum gravity in de Sitter space," hep-th/0106109.

[6] R. Bousso, "Positive vacuum energy and the N-bound," JHEP 0011 (2000) 038 [hepth/0010252]; "Bekenstein bounds in de Sitter and flat space," JHEP 0104 (2001) 035 hep-th/0012052.

[7] A. Volovich, "Discreteness in de Sitter space and quantization of Kaehler manifolds," hep-th/0101176.

[8] A. Chamblin and N. D. Lambert, "De Sitter space from M-theory," Phys. Lett. B 508 (2001) 369 [hep-th/0102159]; "Zero-branes, quantum mechanics and the cosmological constant," hep-th/0107031.

[9] A. Strominger, "The dS/CFT correspondence," hep-th/0106113.

[10] D. Klemm, "Some aspects of the de Sitter/CFT correspondence," hep-th/0106247.

[11] P. O. Mazur and E. Mottola, "Weyl cohomology and the effective action for conformal anomalies," hep-th/0106151]; I. Antoniadis, P. O. Mazur and E. Mottola, "Comment on Nongaussian isocurvature perturbations from inflation," astro-ph/9705200.

[12] M. Li, "Matrix model for de Sitter," hep-th/0106184.

[13] S. Nojiri and S. D. Odintsov, "Conformal anomaly from dS/CFT correspondence," hep-th/0106191; "Quantum cosmology, inflationary brane-world creation and dS/CFT correspondence," hep-th/0107134]; S. Nojiri, S. D. Odintsov and S. Ogushi, "Cosmological and black hole brane world universes in higher derivative gravity," hep-th/0108172].

[14] Y. Gao, "Symmetries, matrices, and de Sitter gravity," hep-th/0107067.

[15] T. Shiromizu, D. Ida and T. Torii, "Gravitational energy, dS/CFT correspondence and cosmic no-hair," hep-th/0109057. 
[16] R. Kallosh, "N = 2 supersymmetry and de Sitter space," hep-th/0109168.

[17] C. M. Hull, "De Sitter Space in Supergravity and M Theory," hep-th/0109213.

[18] M. Spradlin, A. Strominger and A. Volovich, "Les Houches Lectures on De Sitter Space," hep-th/0110007.

[19] S. Perlmutter, "Supernovae, dark energy, and the accelerating universe: The status of the cosmological parameters," in Proc. of the 19th Intl. Symp. on Photon and Lepton Interactions at High Energy LP99 ed. J.A. Jaros and M.E. Peskin, Int. J. Mod. Phys. A 15S1 (2000) 715 [eConfC 990809 (2000) 715].

[20] J. Maldacena and A. Strominger, "Statistical entropy of de Sitter space," JHEP 9802 (1998) 014 gr-qc/9801096.

[21] M. I. Park, "Statistical entropy of three-dimensional Kerr-de Sitter space," Phys. Lett. B 440 (1998) 275 [hep-th/9806119].

[22] M. Bañados, T. Brotz and M. E. Ortiz, "Quantum three-dimensional de Sitter space," Phys. Rev. D 59 (1999) 046002 [hep-th/9807216.

[23] W. T. Kim, "Entropy of $2+1$ dimensional de Sitter space in terms of brick wall method," Phys. Rev. D 59 (1999) 047503 [hep-th/9810169].

[24] F. Lin and Y. Wu, "Near-horizon Virasoro symmetry and the entropy of de Sitter space in any dimension," Phys. Lett. B 453 (1999) 222 [hep-th/9901147.

[25] S. W. Hawking, J. Maldacena and A. Strominger, "DeSitter entropy, quantum entanglement and AdS/CFT," JHEP 0105 (2001) 001 hep-th/0002145.

[26] G. 't Hooft, "Dimensional reduction in quantum gravity," gr-qc/9310026].

[27] L. Susskind, "The World as a hologram," J. Math. Phys. 36 (1995) 6377 hepth/9409089.

[28] J. Maldacena, "The large $N$ limit of superconformal field theories and supergravity," Adv. Theor. Math. Phys. 2 (1998) 231 [Int. J. Theor. Phys. 38 (1998) 1113] thepth/9711200.

[29] R. Bousso, "Holography in general space-times," JHEP 9906 (1999) 028 hepth/9906022.

[30] J. D. Brown and M. Henneaux, "Central charges in the canonical realization of asymptotic symmetries: An example from three-dimensional gravity," Commun. Math. Phys. 104 (1986) 207.

[31] S. Deser and R. Jackiw, "Three-Dimensional Cosmological Gravity: Dynamics Of Constant Curvature," Annals Phys. 153 (1984) 405. 
[32] P. O. Mazur, "Quantum Gravitational Measure For Three Geometries," Phys. Lett. B 262 (1991) 405 hep-th/9701033.

[33] A. Achucarro and P. K. Townsend, "A Chern-Simons Action For Three-Dimensional Anti-De Sitter Supergravity Theories," Phys. Lett. B 180 (1986) 89.

[34] E. Witten, "(2+1)-Dimensional Gravity As An Exactly Soluble System," Nucl. Phys. B 311 (1988) 46.

[35] O. Coussaert, M. Henneaux and P. van Driel, "The Asymptotic dynamics of threedimensional Einstein gravity with a negative cosmological constant," Class. Quant. Grav. 12 (1995) 2961 [gr-qc/9506019].

[36] M. Bañados, K. Bautier, O. Coussaert, M. Henneaux and M. Ortiz, "Anti-de Sitter/CFT correspondence in three-dimensional supergravity," Phys. Rev. D 58 (1998) 085020 hep-th/9805165.

[37] M. Henneaux, L. Maoz and A. Schwimmer, "Asymptotic dynamics and asymptotic symmetries of three-dimensional extended AdS supergravity," Annals Phys. 282 (2000) 31 hep-th/9910013.

[38] M. Rooman and P. Spindel, "Holonomies, anomalies and the Fefferman-Graham ambiguity in AdS(3) gravity," Nucl. Phys. B 594 (2001) 329 hep-th/0008147.

[39] K. Krasnov, "On holomorphic factorization in asymptotically AdS 3D gravity," hepth/0109198.

[40] G. W. Moore and N. Seiberg, "Taming The Conformal Zoo," Phys. Lett. B 220 (1989) 422; S. Elitzur, G. W. Moore, A. Schwimmer and N. Seiberg, "Remarks On The Canonical Quantization Of The Chern-Simons-Witten Theory," Nucl. Phys. B 326 (1989) 108.

[41] A. Alekseev and S. Shatashvili, "Path Integral Quantization Of The Coadjoint Orbits Of The Virasoro Group And 2-D Gravity," Nucl. Phys. B 323 (1989) 719.

[42] M. Bershadsky and H. Ooguri, "Hidden Sl(N) Symmetry In Conformal Field Theories," Commun. Math. Phys. 126 (1989) 49.

[43] P. Forgacs, A. Wipf, J. Balog, L. Feher and L. O'Raifeartaigh, "Liouville And Toda Theories As Conformally Reduced WZNW Theories," Phys. Lett. B 227 (1989) 214.

[44] W. A. Sabra, "Classical Hamiltonian reduction and superconformal algebras," Phys. Lett. B 313 (1993) 68 hep-th/9207012.

[45] N. Seiberg, "Notes On Quantum Liouville Theory And Quantum Gravity," Prog. Theor. Phys. Suppl. 102 (1990) 319. 